\title{
A wide field-of-view microscope based on holographic focus grid
}

\author{
Jigang $\mathrm{Wu}^{*}$, Xiquan Cui, Guoan Zheng, Lap Man Lee, Changhuei Yang \\ Biophotonics Laboratory, Department of Electrical Engineering \\ California Institute of Technology, Pasadena, CA, 91125
}

\begin{abstract}
We have developed a novel microscope technique that can achieve wide field-of-view (FOV) imaging and yet possess resolution that is comparable to conventional microscope. The principle of wide FOV microscope system breaks the link between resolution and FOV magnitude of traditional microscopes. Furthermore, by eliminating bulky optical elements from its design and utilizing holographic optical elements, the wide FOV microscope system is more cost-effective. In our system, a hologram was made to focus incoming collimated beam into a focus grid. The sample is put in the focal plane and the transmissions of the focuses are detected by an imaging sensor. By scanning the incident angle of the incoming beam, the focus grid will scan across the sample and the time-varying transmission can be detected. We can then reconstruct the transmission image of the sample. The resolution of microscopic image is limited by the size of the focus formed by the hologram. The scanning area of each focus spot is determined by the separation of the focus spots and can be made small for fast imaging speed. We have fabricated a prototype system with a $2.4-\mathrm{mm}$ FOV and $1-\mu \mathrm{m}$ resolution. The prototype system was used to image onion skin cells for a demonstration. The preliminary experiments prove the feasibility of the wide FOV microscope technique, and the possibility of a wider FOV system with better resolution.
\end{abstract}

Keywords: Holographic optical element, Scanning microscope, Biomedical imaging

\section{INTRODUCTION}

Conventional microscope usually consists of an objective, or an infinity-corrected objective with tube lens, and an eyepiece for observing the image directly, or lenses to project the image onto a camera. The filed-of-view (FOV) of the microscope can be calculated by the field number divided by the magnification of the objective lens [1]. Thus, there is a trade-off between the FOV and the magnification. And the FOV is usually limited, especially for high magnification objective. For example, for an Olympus PLN 10x objective lens with a numerical aperture of 0.25 and a field number of 22 , the FOV is only $2.2 \mathrm{~mm}$. The requirement for high quality objective lenses also increases the cost for conventional microscope. We notice that in a scanning microscope [2], the field-of-view is determined by the area that the focus light spot can be scanned across, and the resolution is determined by the size of the focus light spot. Using the idea of scanning microscope, if we have multiple focus light spots scanning at the same time, the effective field-of-view will be increased accordingly. We designed and implemented a prototype microscope system using this idea which can achieve wide FOV imaging and still possess resolution that is comparable to conventional microscope. The principle of wide FOV microscope system breaks the link between resolution and FOV of traditional microscopes. In addition, the elimination of expensive objective lenses helps reducing the cost of the imaging system.

Focus array has been used for optical tweezers [3] and can be implemented by various methods, such as focusing mirror array [3], diffractive optical element with an objective lens [4], or microlens array [5], etc. In our wide FOV microscope system, a hologram was made to focus the incoming collimated beam into a focus grid. Compared with other techniques suitable for optical trapping, our hologram can generate a focus grid with wide area, high resolution and a very small spacing between focus spots, which make it suitable for imaging purpose. In the system setup, the focus grid was used to illuminate the sample, and the transmissions of the focuses are detected by an imaging sensor. The scanning of the focuses was achieved by scanning the incident angle of the incoming beam using a scanning mirror. The image of the sample can then be reconstructed. Obviously, the resolution of microscopic image is limited by the size of the focus

\footnotetext{
*jigang@caltech.edu; phone 16263952235
}

Three-Dimensional and Multidimensional Microscopy: Image Acquisition and Processing XVII, edited by Jose-Angel Conchello, Carol J. Cogswell, Tony Wilson, Thomas G. Brown, Proc. of SPIE Vol. 7570, 757009 (c) 2010 SPIE · CCC code: $1605-7422 / 10 / \$ 18 \cdot$ doi: $10.1117 / 12.842303$ 
generated by the hologram and the scanning area of each focus spot is determined by the spacing between the focus spots and can be made small for fast imaging speed.

\section{SYSTEM SETUP}

Two possible methods to record the hologram are shown in Fig. 1. The mask was made by punching small aperture grid on a gold-coated glass slide using focused ion beam. In Fig. 1(a), a beam splitter is used to combined the collimated reference beam and the transmission light through the aperture grid. In this case, the metal layer on the mask will be thick and thus totally opaque. In the recording scheme shown in Fig. 1(b), however, the thickness of the gold layer was chosen so that the laser can transmit through it and attenuated to a certain level. The direct transmission of the laser would serve as the reference beam, and the transmission through the apertures was the sample beam. In both cases, the interference between the reference beam and the sample beam was recorded by a holographic plate. Obviously, the optical distance between the mask and the holographic plate will be the focal length during reconstruction. In our experiment, we chose to use the scheme in Fig. 1(b), and the gold thickness was set to be $200 \mathrm{~nm}$ and the measured optical density was $\sim 4$. Compare the scheme in Fig. 1(a), our inline holography scheme in Fig. 1(b) has several advantages. First, the focal length can be adjusted easily and can be small. The scheme in Fig. 1(a) instead can only set a focal length according to the size of the beam splitter, which cannot be easily made to be small, e.g., less than $1 \mathrm{~mm}$. Second, a large-area aperture grid can be recorded and the numerical aperture of the recording can still be very high. In comparison, the scheme in Fig. 1(b) can only record an area that is limited by the size of the beam splitter, and the numerical aperture is also limited by the size of the beam splitter.

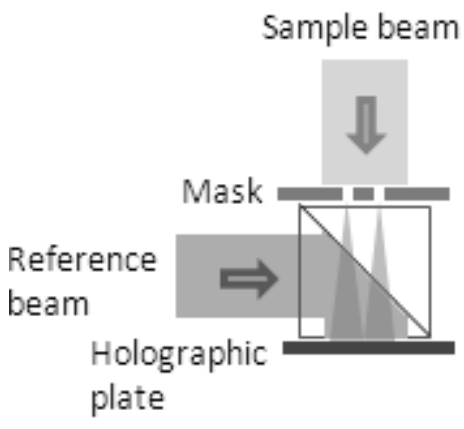

(a)

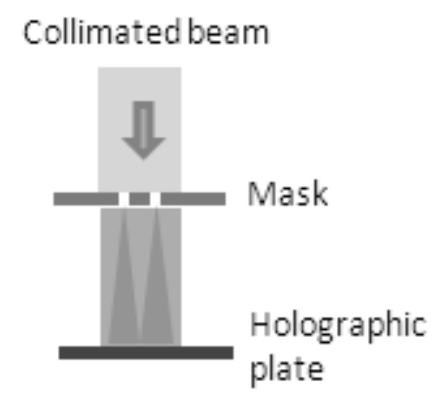

(b)

Fig. 1. Recording scheme for the hologram. (a) A beam splitter combines the collimated reference beam and the transmitted light field through a grid of apertures; (b) The transmitted collimated beam through the thinly-coated metal layer interferes with the transmitted light through the apertures.

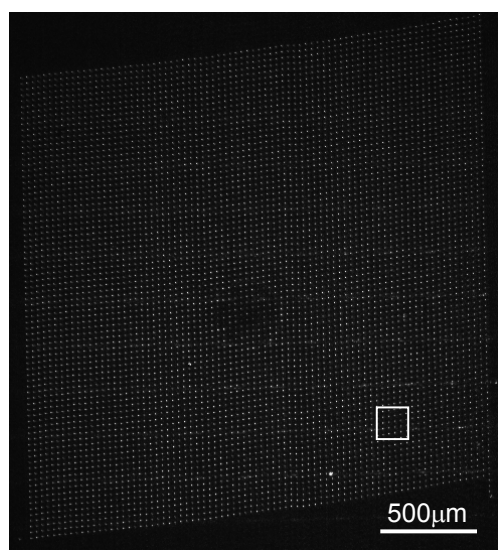

(a)

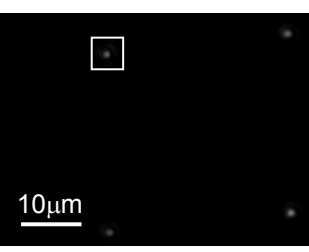

(b)

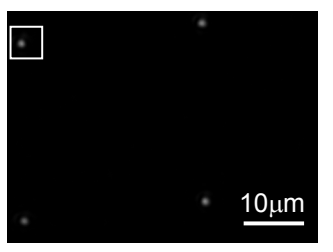

(c)

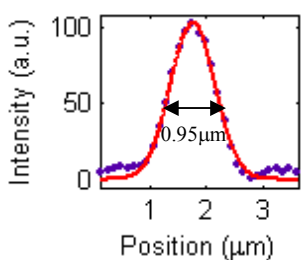

(d)

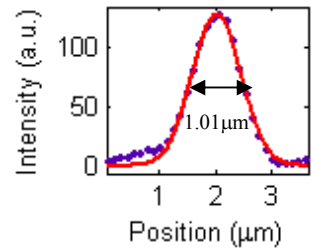

(e)

Fig. 2. (a) Reconstructed focus light spots by a hologram; (b) zoom-in of the spots; (c) the same spots scanned to a different location; (d)(e) measured size of the spots as indicated in (b), (c), respectively. 
The focus grid generated by the hologram that we recorded in the experiment is shown in Fig. 2. Fig. 2(a) shows the focus grid as detected directly by an imaging sensor, where the FOV was around $2.4 \mathrm{~mm}$. Notice that the focus grid was tilted, which was due to hysteresis of the translation stage in the focused ion beam machine. Fig. 2(b)(c) shows the zoom-in of the spots during scanning, where the spacing between focus spots was $30 \mu \mathrm{m}$. Fig. 2(d)(e) shows the measured spot size, which was around $1 \mu \mathrm{m}$. We can see that the spot size didn't change too much during the scanning, so the resolution across the image will be roughly uniform.

After the hologram was recorded, it can be used to build a wide FOV microscope system as shown in Fig. 3. A collimated laser beam was reflected by the scanning mirror and incident on the hologram. The focus grid generated by the hologram then illuminated the sample and the transmission of the focuses was measured using an imaging sensor. The sample was put directly on top of the imaging sensor so that the focus grid plane, which coincide with the sample plane, will be close to the sensor. In this way, the expansion of the focuses on the imaging sensor will be small enough and focus spots can be well separated from one another. A raster scan of the focus grid was perform by the scanning mirror and the image of the sample can then be reconstructed. In our experiment, the wavelength of the laser was 532 $\mathrm{nm}$.

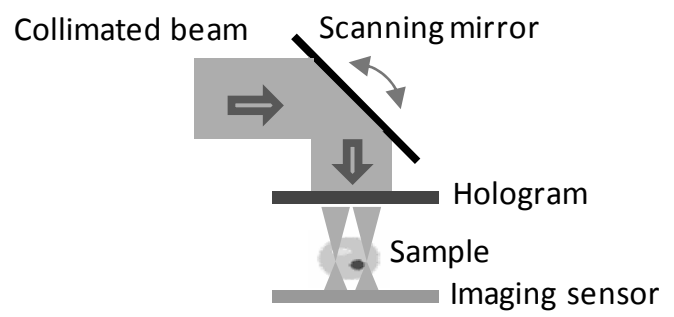

Fig. 3. Wide FOV microscope system. The focus grid is generated by the hologram with incident collimated beam. The collimated beam can be scanned by a scanning mirror and thus the focus grid will be scanned. Image can be obtained by measuring the transmission of each focus beam using an imaging sensor.

\section{IMAGING RESULT}

To demonstrate the resolution of our imaging system, a testing target was imaged, as shown in Fig. 4. The testing target is a transparent bar pattern on an opaque background with dimensions shown in Fig. 4(a). The images of 1- $\mu \mathrm{m}$ bar and $2-\mu \mathrm{m}$ bar are shown in Fig. 4(b) and (c). We can see that the bars can be clearly differentiated, which proved our system resolution of $\sim 1 \mu \mathrm{m}$.

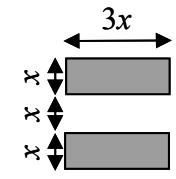

(a)

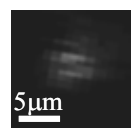

(b)

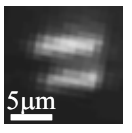

(c)

Fig. 4. (a) Bar pattern target; (b)(c) Images of bars with $x=1 \mu \mathrm{m}$ and $2 \mu \mathrm{m}$, respectively..

The wide FOV system was then used to image onion skin cells, as shown in Fig. 5. The cells and nucleus can be seen in the zoom-in image Fig. 5(b). 


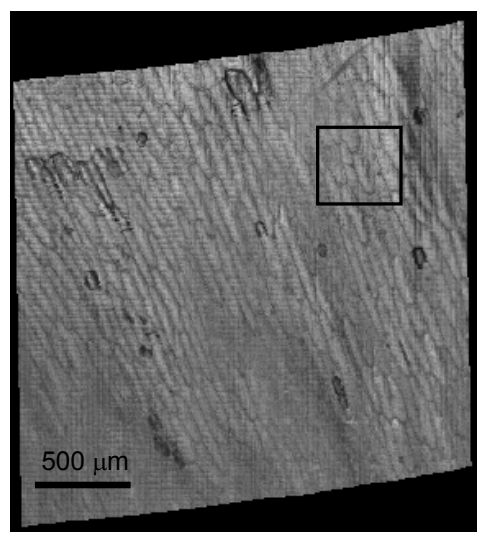

(a)

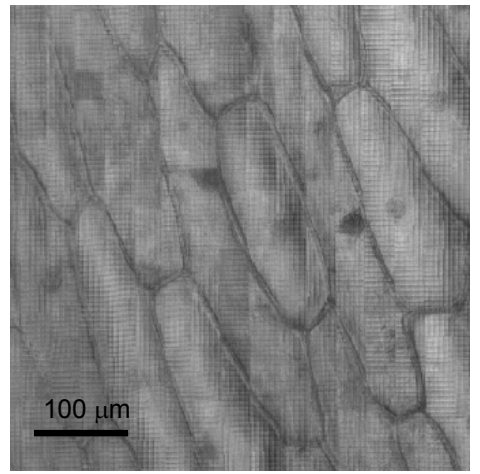

(b)

Fig. 5. (a) Onion skin cell image acquired using the preliminary microscope system; (b) Zoom-in of the region as indicated in (a).

\section{CONCLUSION}

In conclusion, we have developed a novel wide FOV microscope system based on a holographic scanning scheme. The innovation is to use a hologram to generate a focus grid, which was used to illuminate and scan the sample. The transmission through the sample can then be detected and used to reconstruct the image of the sample. We have demonstrated the principle by make a prototype system with 2.4-mm FOV and 1- $\mu \mathrm{m}$ resolution. The prototype system was used to image a testing target and onion skin cells for demonstration. In principle, we can expect to achieve a wider FOV and higher resolution with our scheme.

\section{REFERENCES}

1. http://www.microscopyu.com/articles/formulas/formulasfieldofview.html.

2. T. Wilson, Editor, Confocal Microscopy, Academic Press (1990).

3. F. Merenda, J. Rohner, J. M. Fournier, and R. P. Salathe, "Miniaturized high-NA focusing-mirror multiple optical tweezers," Optics Express 15, 6075-6086 (2007).

4. E. R. Dufresne and D. G. Grier, "Optical tweezer arrays and optical substrates created with diffractive optics," Review of Scientific Instruments 69, 1974-1977 (1998).

5. C. H. Sow, A. A. Bettiol, Y. Y. G. Lee, F. C. Cheong, C. T. Lim, and F. Watt, "Multiple-spot optical tweezers created with microlens arrays fabricated by proton beam writing," Applied Physics B 78, 705-709 (2004). 